\title{
Interstitial engraftment of adipose-derived stem cells into an acellular dermal matrix results in improved inward angiogenesis and tissue incorporation
}

\author{
Issei Komatsu, ${ }^{1}$ Jun Yang, ${ }^{1}$ Ying Zhang, ${ }^{1}$ L. Scott Levin, ${ }^{2}$ Detlev Erdmann, ${ }^{1}$ Bruce Klitzman, ${ }^{1}$ \\ Scott T. Hollenbeck ${ }^{1}$ \\ ${ }^{1}$ Division of Plastic, Reconstructive, Maxillofacial and Oral Surgery, Duke University Medical Center, Durham, North Carolina \\ ${ }^{2}$ Department of Orthopedics, University of Pennsylvania School of Medicine, Philadelphia, Pennsylvania
}

Received 28 June 2012; revised 18 December 2012; accepted 2 January 2013

Published online 2 April 2013 in Wiley Online Library (wileyonlinelibrary.com). DOI: 10.1002/jbm.a.34582

\begin{abstract}
Acellular dermal matrices (ADM) are commonly used in reconstructive procedures and rely on host cell invasion to become incorporated into host tissues. We investigated different approaches to adipose-derived stem cells (ASCs) engraftment into ADM to enhance this process. Lewis rat adipose-derived stem cells were isolated and grafted (3.0 $\times 10^{5}$ cells $)$ to porcine ADM disks $(1.5 \mathrm{~mm}$ thick $\times 6 \mathrm{~mm}$ diameter) using either passive onlay or interstitial injection seeding techniques. Following incubation, seeding efficiency and seeded cell viability were measured in vitro. In addition, Eighteen Lewis rats underwent subcutaneous placement of ADM disk either as control or seeded with PKH67 labeled ASCs. ADM disks were seeded with ASCs using either onlay or injection techniques. On day 7 and or 14, ADM disks were harvested and analyzed for host cell infiltration. Onlay and injection techniques resulted in unique seeding patterns;
\end{abstract}

however cell seeding efficiency and cell viability were similar. In-vivo studies showed significantly increased host cell infiltration towards the ASCs foci following injection seeding in comparison to control group ( $p<0.05$ ). Moreover, regional endothelial cell invasion was significantly greater in ASCs injected grafts in comparison to onlay seeding ( $p<0.05$ ). ADM can successfully be engrafted with ASCs. Interstitial engraftment of ASCs into ADM via injection enhances regional infiltration of host cells and angiogenesis, whereas onlay seeding showed relatively broad and superficial cell infiltration. These findings may be applied to improve the incorporation of avascular engineered constructs. ( $) 2013$ Wiley Periodicals, Inc. J Biomed Mater Res Part A: 101A: 2939-2947, 2013.

Key Words: adipose-derived stem cells, cell engraftment, acellular dermal matrix, angiogenesis

How to cite this article: Komatsu I, Yang J, Zhang Y, Levin LS, Erdmann D, Klitzman B, Hollenbeck ST. 2013. Interstitial engraftment of adipose-derived stem cells into an acellular dermal matrix results in improved inward angiogenesis and tissue incorporation. J Biomed Mater Res Part A 2013:101A:2939-2947.

\section{INTRODUCTION}

Many bioprosthetic materials have been studied for their suitability as matrices for tissue repair and tissue engineering. Collagen-based biomaterials, such as acellular dermal matrix (ADM), have been proposed to be suitable biomaterials. The structural framework and architectural microenvironment found in ADM are favorable for cell incorporation after implantation. ${ }^{1-4}$ The materials can also be chemically cross-linked to increase the mechanical stability. ${ }^{5}$ The combination of these characteristics make ADM an appealing material for clinical application.

Non-cross-linked ADM may be limited in its utility due to lower durability in clinical setting, such as abdominal wall hernia. ${ }^{6,7}$ Cross-linked ADM, on the other hand, has the potential for a broad range of applications in plastic and reconstructive surgery as it offers long-lasting dimensional stability that ensures the integrity of the collagen graft during the wound-healing process. ${ }^{2,6,8}$ However, the strength advantage associated with collagen crosslinking may limit tissue incorporation through collagen degradation and vascular ingrowth. ${ }^{1,5}$ Factors which promote a rapid degree of host cell infiltration and angiogenesis within cross-linked acellular constructs may enhance biocompatibility.

A large body of literature has shown that mesenchymal stem cells, such as adipose-derived stem cells (ASCs), possess developmental plasticity both in vitro and in vivo and may be an ideal cell source for therapeutic angiogenesis and tissue engineering. ${ }^{9-11}$ ASCs are easily harvested and have been shown to produce physiologically relevant levels of multiple angiogenic and anti-appoptotic growth factors with simultaneous self-differentiation to mature cell lineages. $^{9-12}$ 
Based on these observations, we chose to determine whether pluripotential ASCs could be used to enhance cell infiltration and angiogenesis in collagen cross-linked ADM constructs. Previous studies showed that ACSs would successfully attach to and proliferate on ADM. ${ }^{13,14}$ Importantly, these reports indicates seeding with ASCs to ADM enhanced tissue incorporation through cell infiltration and angiogenesis. However, these studies employed surface application of ASCs on ADM and would have limited use in inducing deep cell ingrowth and vascularization into biomaterial. We thus hypothesized that seeding the ASCs within the interstitium of the ADM construct, rather than on the surface, would enhance angiogenesis and cell infiltration throughout the material. The feasibility of ADM engraftment with ASCs is tested as a primary step toward delivery of biologically active constructs.

\section{METHODS}

Acellular dermal matrix preparation

Cross-linked ADMs were used throughout the study as the construct (Permacol ${ }^{\mathrm{TM}}$, Covidien, Mansfield, MA). The ADM is processed from harvested porcine dermis by removing all cellular elements with organic solvents followed by enzymatic extraction of all genetic material. The resulting acellular collagen matrix consisting of Types I and III collagen and elastin is then cross-linked for enhanced durability. The material used was provided in a large sheet form $(16 \times 10$ $\mathrm{cm}^{2}, 1.5 \mathrm{~mm}$ thick) and cut into $6 \mathrm{~mm}$ disks for implantation. There is no difference in the two surfaces of ADM.

\section{Cell isolation and differentiation}

All animal studies were performed in conjunction with the Duke University IACUC. NIH guideline for the care and use
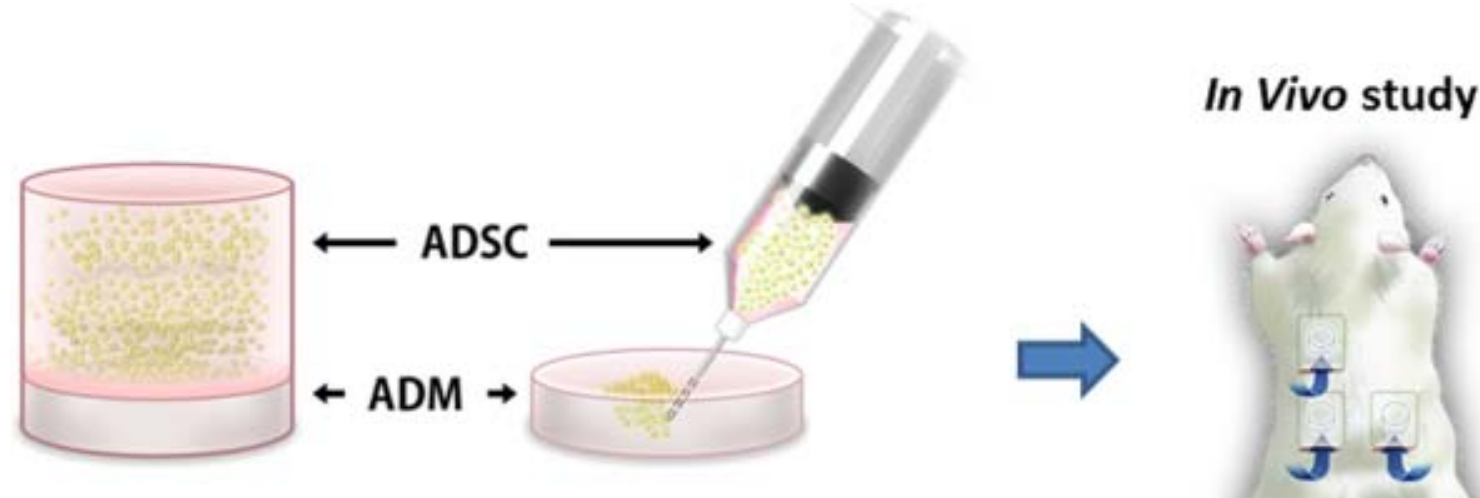

\section{Onlay Seeding}

Injection Seeding

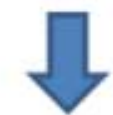

In Vitro study

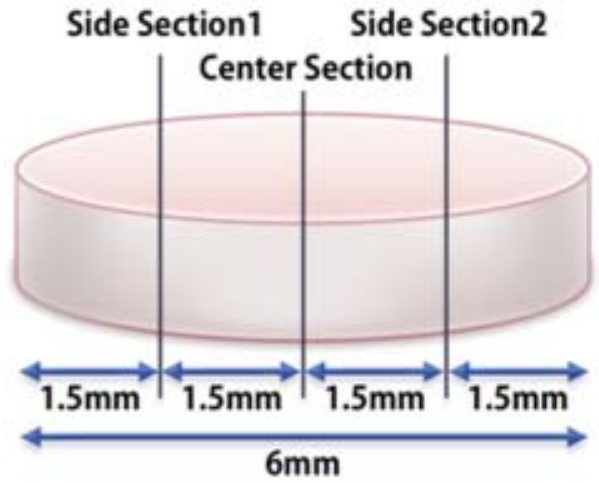

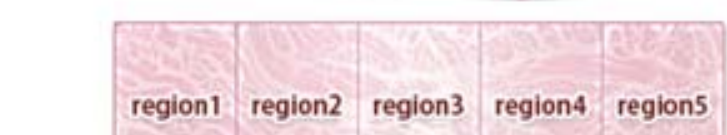
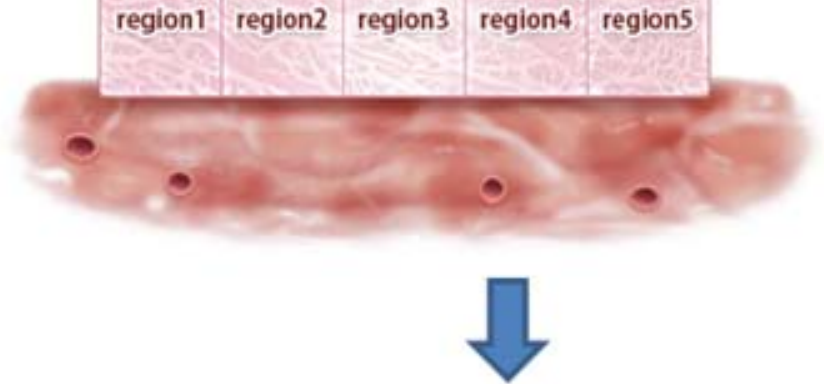

Image and Date analysis

FIGURE 1. Flowchart of ASCs engraftment to ADM, in vitro and in vivo study. ASCs were grafted to ADM disks using either passive onlay or interstitial injection seeding technique (above left). In in vitro study, seeding efficiency and seeded cell viability were measured in three crosssections of ADM (below left). In in vivo studies, Lewis rats underwent subcutaneous placement of ADM disks seeded with PKH-labeled ASCs using either onlay or injection technique (above right). On day 7 and or 14, the disks were harvested and analyzed for host cell and endothelial cell infiltration. To evaluate regional cell infiltration toward ADM, images of all cross-sectioned ADM disks were divided into five rectangular regions, and each region was analyzed individually (below right). [Color figure can be viewed in the online issue, which is available at wileyonlinelibrary.com.] 
of laboratory animals (NIH Publication \#85-23 Rev.1985) has been observed. Adult rat ASCs were isolated from the inguinal fat pad of syngeneic male adult Lewis rats (Charles River Laboratories, Wilmington, MA) through collagenase digestion and centrifugation as described previously. ${ }^{15}$ For in vivo studies, the cultured ASCs were labeled with the green fluorescent cell linker, PKH-67 $\left(2 \times 10^{-7} \mathrm{M}\right.$, SigmaAldrich, Saint Louis, MO) prior to engraftment to distinguish ASCs from host cells.

\section{Cell seeding}

ADM disks (1.5 mm thick $\times 6 \mathrm{~mm}$ diameter) were washed with PBS for $5 \mathrm{~min}$. Onlay seeding was performed by incubating with $3.0 \times 10^{5}$ ASCs as described in previous reports. ${ }^{13,16}$ Briefly, $6 \mathrm{~mm}$ ADM disks were placed on the bottom of 96-well plates and a concentrated cell solution $\left(3.0 \times 10^{5}\right.$ cells $/ 300 \mu \mathrm{l}$ of DMEM) was added on the top of the ADM disks. Injection seeding was performed by injecting the concentrated cell solution $\left(3.0 \times 10^{5}\right.$ cells $/ 300 \mu \mathrm{l}$ of DMEM) into the center of the disk using a 25-gauge needle, following previously described methods. ${ }^{17}$

\section{Semi-quantitative analysis of cell seeding efficiency}

At 7 days post-incubation, ADM disks from both onlay and injection seeding techniques were washed in PBS. $(n=12$, each group) Among them, six ADM disks from each seeding technique ( $n=3$, each group) were immersed in $10 \%$ formalin, paraffin-fixed, and cut into $5 \mu \mathrm{m}$ sections at center section of disk for hematoxylin and eosin (H\&E). For the remaining ADM disks ( $n=9$, each group), three crosssections of disks were evaluated; side section1, center section, and side section2 (Fig. 1). These samples were then cryosectioned at $10 \mu \mathrm{m}$. Slides were stained with 4,6-6iamidino-2-phenylindole(DAPI) $(600$ nM, Sigma-Aldrich, St. Louis, MO) using standard methods. Representative sections from the three regions were analyzed using a widefield fluorescence microscope (Carl Zeiss Microscopy, Thornwood, NY). The total number of cells stained positive with DAPI was determined and counted using Image J software (Public domain image processing program, National Institutes of Health, Bethesda, MD).

\section{Live/dead cell analysis}

Live and dead cell analysis was assessed using live stain (carboxyl fluorescein diacetate, succinimidyl ester (CFDASE)) (2.5 mM, Invitrogen, Carlsbad, CA). At $6 \mathrm{~h}$ post-incubation, ADM disks from each seeding technique $(n=6$, each group) were embedded and sectioned. Samples were stained with CFDA-SE and DAPI. A representative center section of each disk in each seeding technique was analyzed. The percentage of live and dead cells were counted and determined by following formulas: Percentage of Live cells $=$ total numbers of live cells in cell-seeded ADM (CFDA-SEpositive cells)/total number of cells in cell-seeded ADM (DAPI-positive cells)

Percentage of dead cells $=100-$ percentage of live cells

\section{Surgical procedure: engraftment of cell-seeded ADMs} and control

Eighteen male adult Lewis rats were grafted with three different disks: control, ADM disks with ASCs onlay seeding, and ADM disks with ASCs injection seeding. At $6 \mathrm{~h}$ postincubation, ADM disks from each seeding technique were rinsed in PBS and inserted into dorsal subcutaneous pockets through a $10 \mathrm{~mm}$ incision (Fig. 1). For onlay seeded ADM disks, the engrafted surface was placed toward the base of the subcutaneous pocket. For injection seeded ADM disks, the needle path away from the base of the subcutaneous pocket. Nine rats were sacrificed at post-implantation day 7 and day 14. Each disk was harvested including a $1 \mathrm{~cm}$ rim of subcutaneous tissues at the base of the pocket.

\section{Immunohistochemisty}

Explanted ADM disks were immersed in 10\% formalin, paraffin-fixed, and cut into $5 \mu \mathrm{m}$ sections at center section of disk for hematoxylin and eosin (H\&E) ( $n=3$, each group). Remaining ADM disks ( $n=6$, each group) were rinsed in PBS and embedded with OCT and snap frozen in liquid nitrogen. ADM disks were cross-sectioned at the center to achieve 40 $\mu \mathrm{m}$ serial sections. Engrafted ASCs were identified within the explant on the basis of green fluorescence of PKH-67 cell linker. Sections were fixed in acetone for $15 \mathrm{~min}$ and then incubated with primary antibodies. For detecting microvessel density, mouse-anti rat CD31 monoclonal antibody (1:100) (BD Biosciences Pharmingen, San Diego, CA) was utilized. Cy5-conjugated affinipure donkey anti-mouse IgG (1:100) (Jackson ImmunoResearch laboratories, West grove, PA) was used as a secondary antibody for evaluation of CD31. Samples were then stained with DAPI. Negative controls were defined as the omission of the primary antibody in the same micro slides section.

\section{Image analysis}

Whole sections were captured using a monochromatic camera under a confocal microscope (Leica Microsystems, Bannockburn, IL). To investigate the effect of seeding techniques on cell infiltration and endothelial cell infiltration, Image J software was used to measure average gray-scale intensity for images stained with DAPI, PKH-labeled ASCs, and CD31. To evaluate regional cell infiltration toward interstitium of ADM construct, images of all cross-sectioned ADM disks were divided into five rectangular regions evenly, and each region was analyzed individually. All pixels representing ADM construct (background) were excluded (set to 0) using a threshold function to identify stained cells (fluorescent intensity 0149). Thereafter, a complete 16-bit histogram was recorded (fluorescent intensity 0-255) and values of grey-scale intensity of cells either within ADM disks or each region ware recorded. To accurately assess host cell infiltration toward $\mathrm{ADM}$, grey-scale intensity of DAPI-positive cells was subtracted by that of PKH-positive cells (grafted ASCs) either in each region or the entire ADM construct area.

\section{Statistical analysis}

Values are presented as means \pm standard deviation. All analysis was performed using the Statistical Program for 


\section{Onlay}
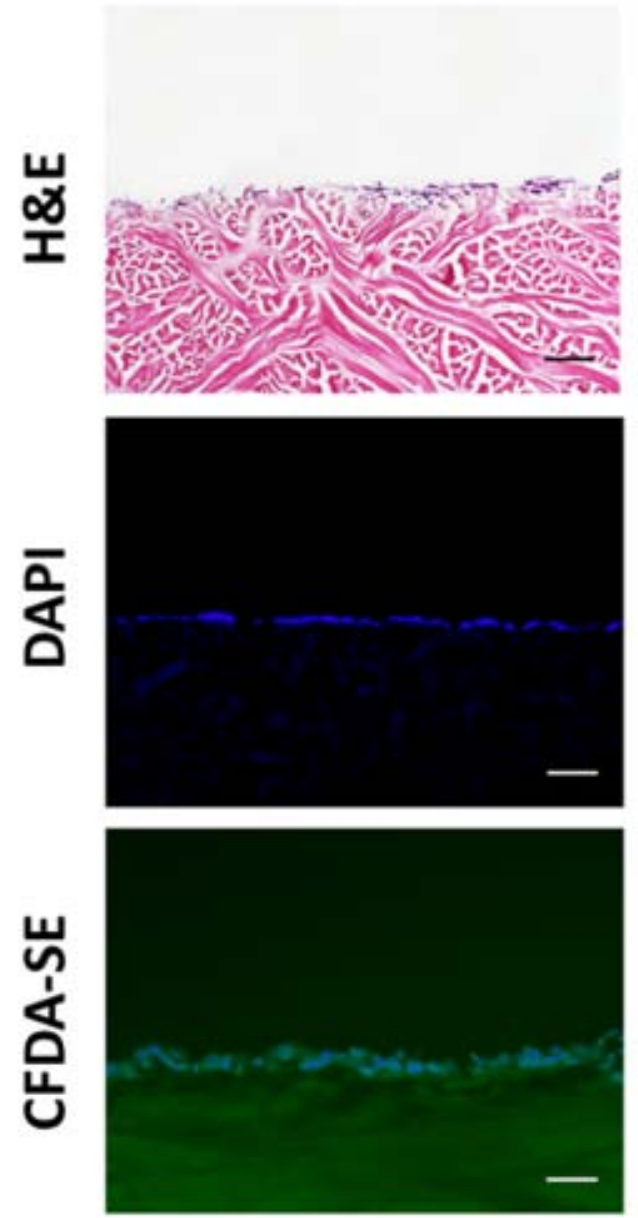

Injection
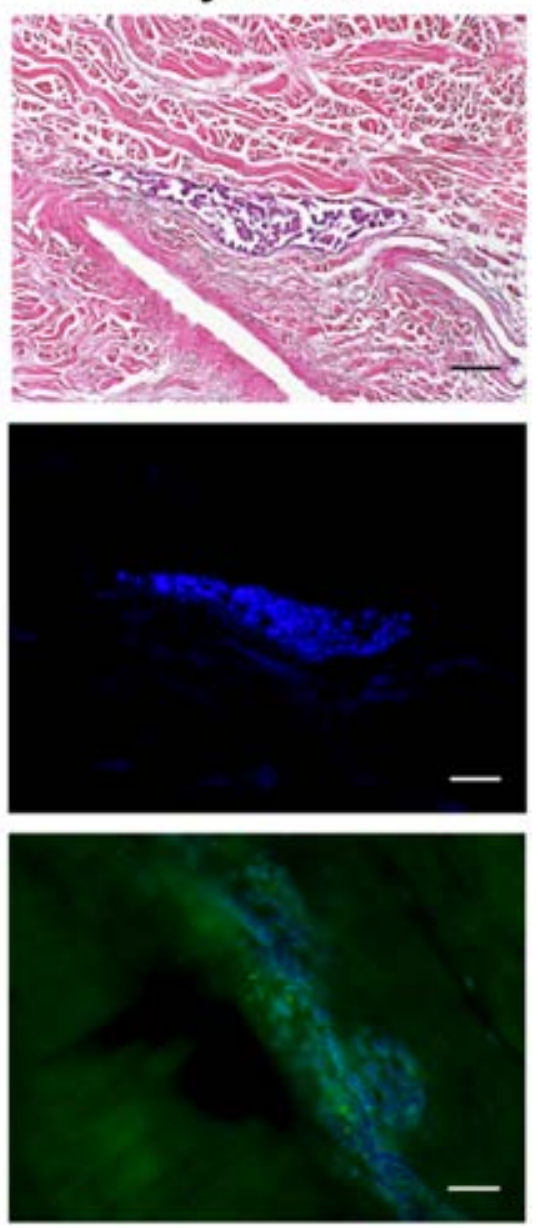

FIGURE 2. In vitro ASCs engraftment. Representative images of H\&E and DAPI staining of ADM disks 7 days after engraftment with ASCs (above and middle row) (scale bars $=100 \mu \mathrm{m}$ ). Both onlay and injection techniques resulted in successful engraftment of ASCs within the ADM. Representative images of DAPI (blue) and CFDA-SE (green) staining $6 \mathrm{~h}$ after engraftment with ASCs (below) (scale bars $=50 \mu \mathrm{m}$ ). Co-localized viable cells appear in both onlay and injection techniques. [Color figure can be viewed in the online issue, which is available at wileyonlinelibrary.com.]
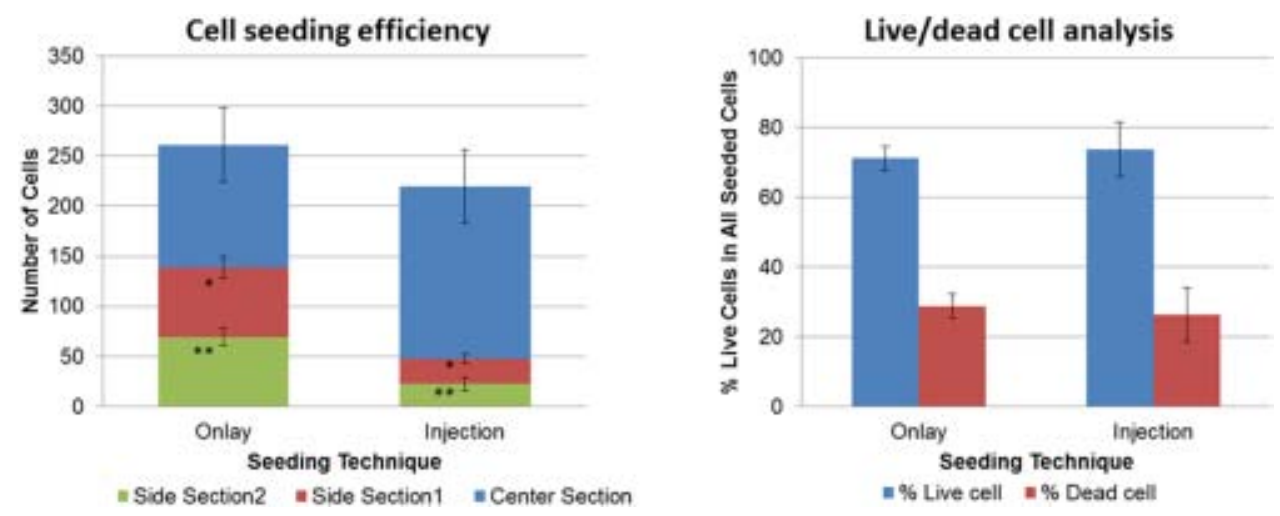

FIGURE 3. Cell seeding efficiency and live/dead cell analysis. Mean total number of cells found within three sections of ADM disks following onlay and injection seeding of ASCs (left). In both techniques, ADM disks were seeded with a cell density of $3.0 \times 10^{5}$ cells and processed with DAPI staining. No significant difference in total cell number and center section $(p>0.05)$. A significant difference was noted in side section 1 and 2 (single and double asterisks indicate $p<0.05$ ). Mean percentage of live and dead cells associated with ADM disks $6 \mathrm{~h}$ after seeding using DAPI and CFDA-SE staining (right). No significant difference between two techniques $(p>0.05)$. [Color figure can be viewed in the online issue, which is available at wileyonlinelibrary.com.] 
Social Science (SPSS, Chicago, IL). For independent samples, students $t$-test was performed with equal variances not assumed to determine significance of all data. A value of $p<0.05$ was considered to define statistical significance.

\section{RESULTS}

\section{Comparison of cell seeding methods}

Both onlay and injection techniques resulted in successful engraftment of ASCs within the ADM disks: confirmed by H\&E, DAPI, and CFDA-SE staining (Fig. 2). The onlay technique resulted in a dense layer of cells on the margin of the ADM disks with a few cells entering the deeper interstices of the construct. The injection technique resulted in cell delivery to foci within the center of the construct.

\section{Cell seeding efficiency}

The ASCs seeding efficiency of each engraftment method was determined by analysis of DAPI-stained sections (Fig. 3, left). Despite the similarity in total seeding efficiency, the overall distribution of cells within the construct was significantly different $(263 \pm 28.6$ versus $220 \pm 21.9$; onlay and injection for total cell number, respectively, $(p>0.05), 122$ \pm 13.4 versus $172 \pm 21.6$; onlay and injection at center section, respectively ( $p>0.05), 69 \pm 7.6$ versus $25 \pm 6.6$; onlay and injection at side section 1 , respectively $(p<$ $0.05), 71 \pm 7.6$ versus $22 \pm 21.9$; onlay and injection at side section 2 , respectively $(p<0.05)$. ADM disks engrafted via injection seeding technique had a larger proportion of ASCs within the center of the construct. This shows that ASCs are effectively delivered to different sites within the construct dependent on the seeding technique used.

Next, we wished to determine the effects of the specific seeding technique on cell viability. At $6 \mathrm{~h}$ post engraftment, ADMs were stained with CFDA-SE to determine ASCs viability. Both techniques resulted in $>70 \%$ cell viability $(71.2 \pm$ $3.58 \%$ versus $73.8 \pm 7.8 \%$; onlay and injection seeding, respectively ( $p>0.05)$ ) (Fig. 3, right). Overall, these findings suggest that both techniques result in effective ASCs engraftment into ADM constructs. In contrast, cell distribution is markedly different, with onlay techniques resulting in diffuse peripheral seeding and injection techniques resulting in focally dense inner construct seeding.

\section{Total and regional host cells infiltration}

To determine whether ASCs engraftment would affect ADM incorporation, we analyzed total and regional host cell infiltration toward ADM constructs. Explanted ADM disks stained with $\mathrm{H} \& \mathrm{E}$ revealed total cellular presence in both onlay and injection seeding group (Fig. 4). Both mononuclear and polynuclear cells were foremost accumulated in the interface in onlay seeding group but could also be found at interstitium of ADM construct in injection seeding group. Capillary structures were observed around ASCs injection spot in injection group. In immunohistochemistory, total cellular presence was observed in DAPI staining (Fig. 5 second column from left). PKH-labeled ASCs were successfully identified in both onlay and injection seeding groups post explantation (Fig. 5. center column, middle and below). The distribution of engrafted ASCs using both methods was similar to that observed in vitro. Control groups had little cellular invasion and a modest cellular response along the margin (Fig. 5. above). ADMs engrafted via the onlay seeding technique demonstrated PKH-labeled ASCs along the margin of the ADM in association with a strong host cellular response (Fig. 5. middle row). ADM engrafted with ASCs through the injection technique showed host cells migrating toward the ASCs injection spot (Fig. 5. below). This focal infiltration is in contrast to the relatively broad and superficial infiltration of onlay seeding group and scant infiltration of the control group.

\section{Quantitative analysis of cell Infiltration}

Both techniques of ASCs engraftment resulted in a significantly greater number of total infiltrated cells within the ADM at 1 and 2 weeks post explantation in comparison to controls $\left(2984.5 \pm 396.5 \mathrm{~m}^{2}, 14637.5 \pm 3132.6 \mu \mathrm{m}^{2}, 18064.8 \pm\right.$ $2435.4 \mu \mathrm{m}^{2}$, in control, onlay and injection at 1 week, respectively $(p<0.05), 9007.5 \pm 2416.3 \mu \mathrm{m}^{2}, 27779.8 \pm 1677.6$ $\mu \mathrm{m}^{2}, 36457.6 \pm 4992.9 \mu \mathrm{m}^{2}$, in control, onlay seeding and
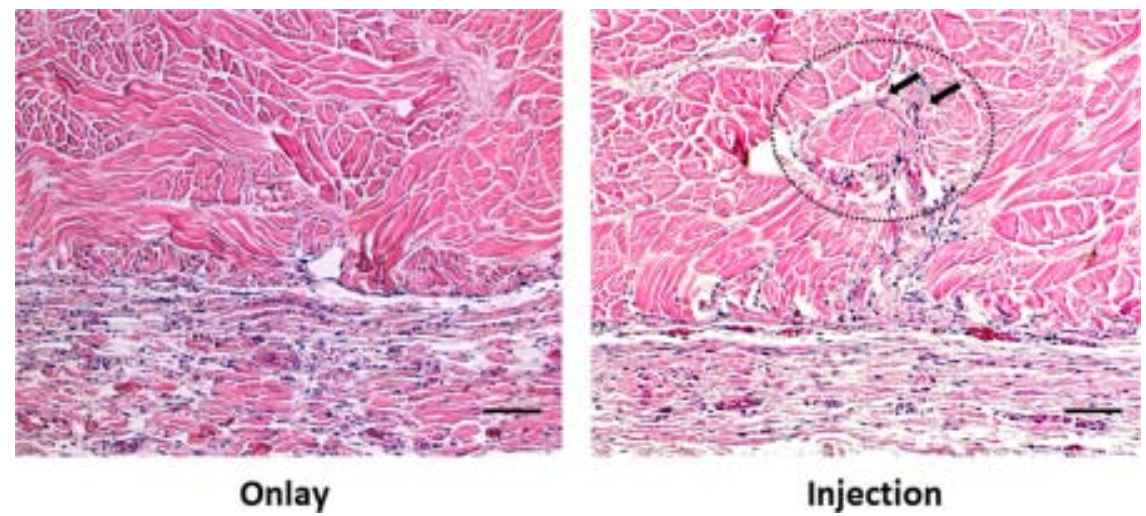

FIGURE 4. Representative H\&E images of ADM disks explanted after 2 weeks of subcutaneous incubation in rat dorsal skin. ADM engrafted with ASCs using an injection technique (right) showed the cellular presence around injection spot (circle), whereas ADM engrafted with ASCs using an onlay technique (left) showed relatively superficial cellular presence. Note that capillary structures were seen in injection group (arrows) (scale bars $=100 \mu \mathrm{m}$ ). [Color figure can be viewed in the online issue, which is available at wileyonlinelibrary.com.] 


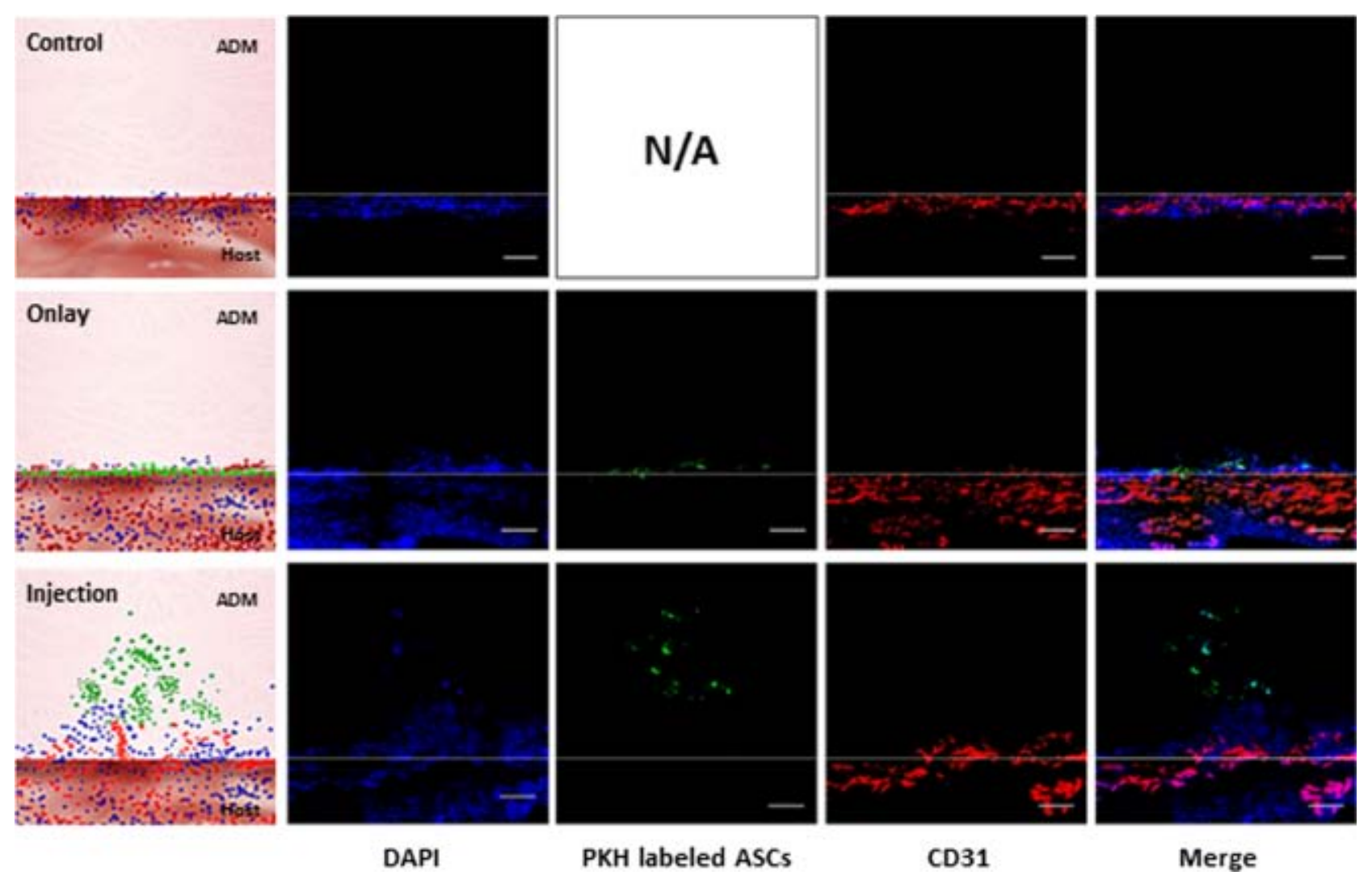

FIGURE 5. Representative immunohistochemistry images of ADM disks explanted after 2 weeks of subcutaneous incubation in rat dorsal skin. Disks were either not engrafted with ASCs (control; above), engrafted with ASCs using onlay technique (middle row), engrafted with ASCs using the injection technique (below). Schematic illustrations are displayed on the left column. Total cell infiltration was evaluated with DAPI staining (second column from left). ADM disks were analyzed with green fluorescence to evaluate PKH labeling of ASCs (center column). Immunohistochemistry was performed to assess CD-31-positive cells (second column from right). DAPI, PKH fluorescence, and CD-31 staining merged (right column). PKH-labeled ASCs were successfully identified in both onlay and injection seeding groups. Total cellular presence and endothelial cell infiltration were more intense in the injection seeding group. Relatively superficial infiltration was seen in onlay seeding group and control group. White dash lines represent the interface between the host and the ADM construct. Scale bars $=100 \mu \mathrm{m}$. [Color figure can be viewed in the online issue, which is available at wileyonlinelibrary.com.]

injection at 2 weeks, respectively $(p<0.05)$ ) (Fig. 6, above left and above right). At 1 week and 2 weeks post implantation ADMs engrafted with ASCs using either onlay or injection seeding showed no significant difference regarding a total number of infiltrated host cells $(p>0.05)$.

For regional assessment, there were multiple differences noted between the engraftment techniques. Along the periphery (region 1 and region 5), there was no significant difference between two techniques in cell infiltration at 1 or 2 weeks. Within the central of the ADM (region 3), there was a trend toward increased cell infiltration in the injection group at 1 week and a significant difference at 2 weeks post implantation $\left(165.5 \pm 14.4 \mu^{2}, 479.8 \pm 188.2 \mu \mathrm{m}^{2}, 1716.3 \pm\right.$ $230.7 \mu \mathrm{m}^{2}$, in control, onlay and injection at region 3 at 1 weeks respectively $(p<0.05), 1734.8 \pm 560.4 \mu \mathrm{m}^{2}, 5092.1$ $\pm 727.3 \mu \mathrm{m}^{2}, 10606.8 \pm 2032.8 \mu \mathrm{m}^{2}$, in control, onlay and injection at region 3 at 2 weeks, respectively $(p<0.05)$ ) (Fig. 6 , below left and above right). Overall, this suggests that total ADM cell infiltration is enhanced by engraftment with ASCs. Moreover, injection of the ASCs into the central portion of the ADM will produce a focal and more intense infiltrative effect.
Total and regional endothelial cells infiltration

Injection seeding resulted in a significantly greater number of CD31-positive cells within the ADM at 1 and 2 weeks post implantation in comparison to controls (911.8 \pm 67.0 $\mu \mathrm{m}^{2}$ versus $4556.5 \pm 384.2 \mu \mathrm{m}^{2}$; control and injection at 1 week, respectively ( $p<0.05$ ), $2574.0 \pm 322.2 \mu \mathrm{m}^{2}$ versus $1068.8 \pm 1988.4 \mu^{2}$; control and injection at 2 weeks, respectively $(p<0.05)$ ). Moreover, total CD31-positive cell infiltration was significantly different between onlay and injection seeding at 1 week $(p<0.05)$, but not at 2 weeks post explantation $(p>0.05)\left(2006.8 \pm 474.4 \mu \mathrm{m}^{2}\right.$ versus $4556.5 \pm 384.2 \mu \mathrm{m}^{2}$; onlay and injection at 1 week, respectively $(p<0.05), 8091.6 \pm 974.3 \mu \mathrm{m}^{2}$ versus $1068.8 \pm$ $1988.4 \mu^{2}$; onlay and injection at 2 weeks, respectively $(p>0.05)$ ) (Fig. 7, above right and above left).

For regional assessment, CD31-positive cell infiltration of ASCs-injected ADM at region 3 was significantly greater in comparison to control and onlay seeding at 1 week and 2 weeks post explantation $\left(166.5 \pm 14.4 \mu \mathrm{m}^{2}, 479.8 \pm\right.$ $188.2 \mu \mathrm{m}^{2}, 1716.3 \pm 230.7 \mu \mathrm{m}^{2}$ in control, onlay, and injection at 1 week respectively $(p<0.05) 589.0 \pm 84.2 \mu \mathrm{m}^{2}$, 
Total host cells infiltration (1week)

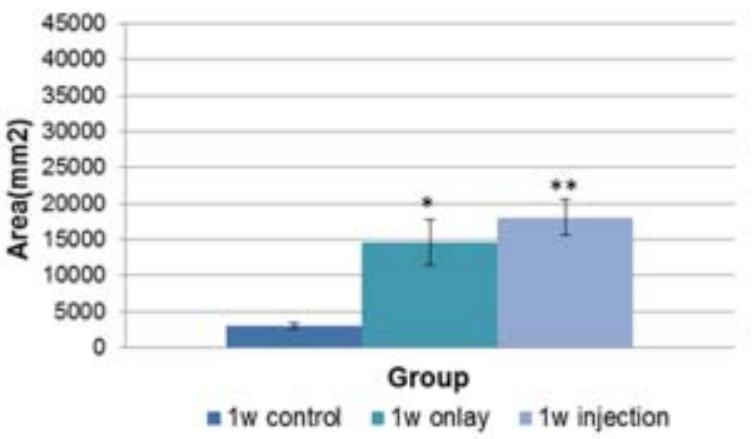

Host cell infiltration (1week)

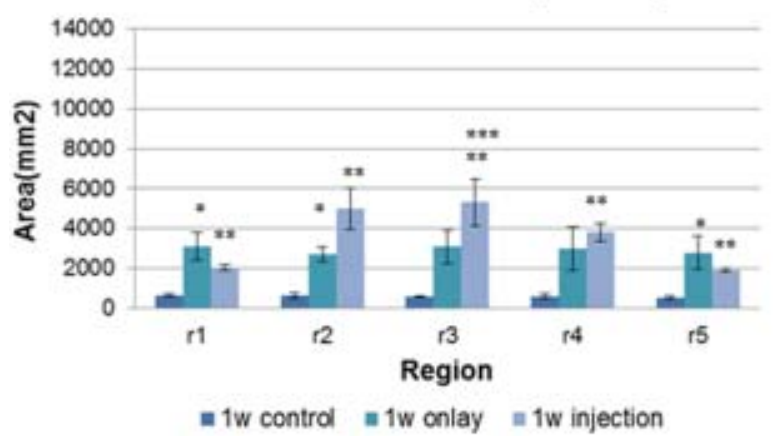

Total host cells infiltration (2week)
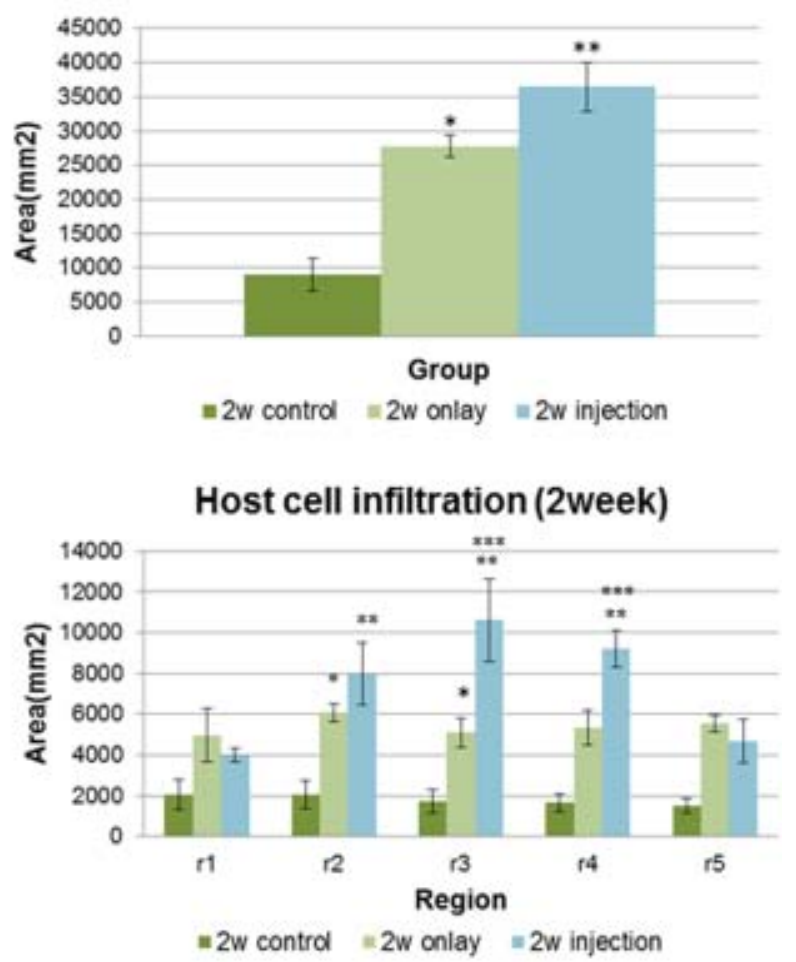

FIGURE 6. Mean total and regional host cell infiltration toward ADM at 1 week and 2 weeks post explantation. Both techniques of ASCs engraftment resulted in a significantly greater number of total infiltrated cells within the ADM at 1 and 2 weeks post explantation in comparison to controls (above left and above right). For regional assessment, there was a trend toward increased cell infiltration within the central of the ADM (region 3) in the injection group (below left and below right). Single and double asterisks indicate $p<0.05$ versus control group. Triple asterisks indicate $p<0.05$; onlay versus injection. Error bars $=\mathrm{SD}$. [Color figure can be viewed in the online issue, which is available at wileyonlinelibrary.com.]

$1661.0 \pm 340.6 \mu \mathrm{m}^{2}, 4084.3 \pm 712.4 \mu \mathrm{m}^{2}$ in control, onlay, and injection at 2 weeks respectively $(p<0.05)$ ) (Fig. 7 , below right and below left). These findings demonstrated that injection of the ASCs into ADM enhances early overall angiogenesis and provides the capacity for focal dense angiogenesis.

\section{DISCUSSION}

Acellular scaffolds are now routinely used in reconstruction of human tissue defects. One of the most significant challenges in this area remains host incorporation and vascularization of the construct. In this study, we have sought to improve host cell infiltration and angiogenesis within a cross-linked ADM by engrafting non-immune mesenchymal stem cells prior to implantation. We proposed that ASCs seeded into the construct would promote host cell invasion and angiogenesis. In addition to improving incorporation, seeding acellular constructs with ASCs may be an effective means for targeted in vivo cell delivery and also be a way to direct in situ remodeling of the grafted construct and surrounding tissues. ${ }^{13,18}$

In the presented study, we first highlighted the feasibility and effectiveness of the two seeding techniques, onlay and injection. Despite the difference in seeding techniques, the results of the in vitro study showed that both methods had similar cell seeding efficiency and viability. After $6 \mathrm{~h}$ of cell incubation, onlay seeding resulted in a dense layer of viable cells on the outer surface of scaffold, which is consistent with the previous studies. ${ }^{13,17,18}$ Injection seeding also showed successful cell delivery. However, the injection technique resulted in a dense cell collection near the center region. This result suggests that both seeding methods can be a feasible approach for cell engraftment onto surgical biomaterials.

Onlay seeding has been the most commonly used seeding method in tissue engineering because of its ease of application. ${ }^{13,17,18}$ Recent reports have suggested that the post-engraftment mechanical performance of ADM and adjacent wound healing could be enhanced by onlay seeding. ${ }^{13,19,20}$ When cell-seeded ADM were implanted into rat subcutaneous tissue, we found that, at 2 weeks, both ADM injected with ASCs and ADM coated with ASCs became more rapidly and thoroughly infiltrated by host cells than control. More importantly, ADM injected with ASCs became more robustly and focally infiltrated by both host cells and endothelial cells toward the vicinity of the ASCs injection spot, in comparison to relatively broad and superficial infiltration of ADM coated with ASCs. These findings indicate that this occurs as a result of chemotactic signals originating from within the matrix, rather than from the ADM surface.

In fact, previous reports demonstrated that ASCs secrete multiple angiogenic and antiapoptotic growth factors, such as VEGF, FGF-2, and HGF. ${ }^{91,21}$ Since the proliferation of 
Total CD31 positive cell infiltration (1week)

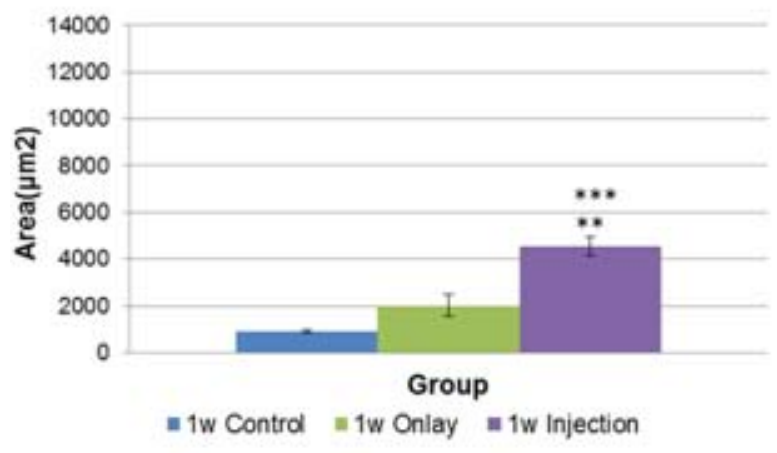

CD31 positive cell infiltration (1week)

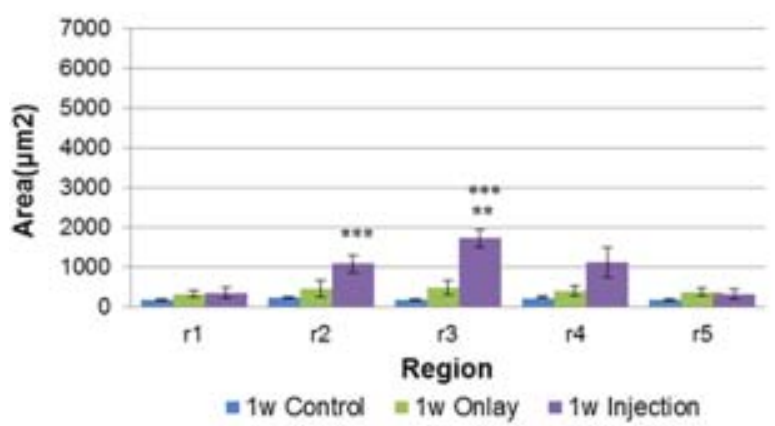

Total CD31 positive cell infiltration (2week)

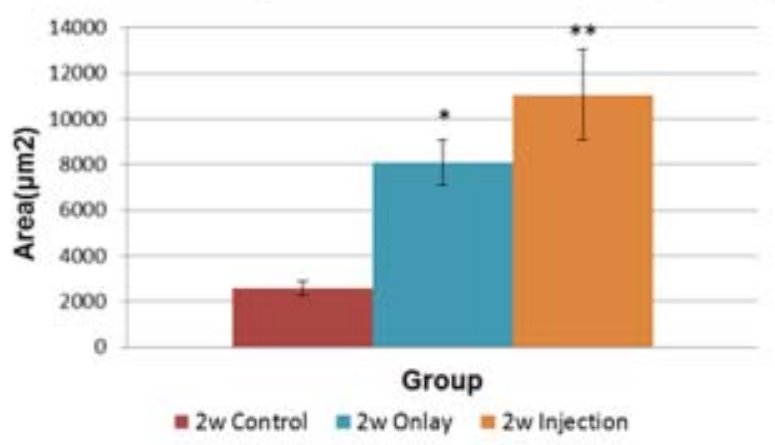

\section{CD31 positive cell infiltration (2week)}

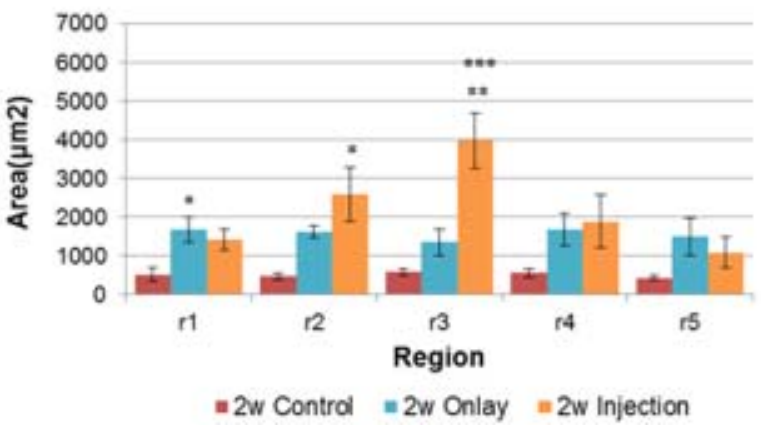

FIGURE 7. Mean total and regional CD31-positive cell infiltration toward ADM at 1 week and 2 weeks post explantation. Both onlay and injection seeding resulted in a significantly greater number of CD31-positive cells within the ADM at 2 weeks post explantation in comparison to controls $(p<0.05)$ (above right and above left). For regional assessment, CD31-positive cell infiltration of ASCs-injected grafts at region 3 was significantly greater in comparison to control and onlay seeding at 1 week and 2 weeks post explantation $(p<0.05)($ below right and below left). Single and double asterisks indicate $p<0.05$ versus control group. Triple asterisks indicate $p<0.05$; onlay versus injection. Error bars $=\mathrm{SD}$. [Color figure can be viewed in the online issue, which is available at wileyonlinelibrary.com.]

endothelial cells and the inhibition of their apoptosis is critical for the growth of new blood vessels, ${ }^{22}$ the secretion of these growth factors by ASCs is likely to promote angiogenesis from the surrounding tissue. The additional ability of ASCs to respond to a stimulus such as hypoxia could allow them to adapt to the environment into which they are placed, by modulating the production of VEGF in response to ischemia. ${ }^{9}$ The hypoxia response appears to be a powerful source of ASCs growth factor secretion and may also be important in regulating angiogenesis within adipose tissue. ${ }^{9,23}$ These findings suggest onlay seeding of ASCs would have limited use in inducing deep cell ingrowth and vascularization into biomaterial. In the present study, we utilized this principle to generate blood vessel growth into an acellular construct.

The compliance and tensile strength of ADM can be altered by the process of collagen cross-linking. The previous study has shown that cross-linked ADMs can provide a strong and durable repair for up to 6 months in abdominal wall hernia models in comparison to loss of tensile strength associated with marked thinning observed in non-crosslinked ADM. ${ }^{7}$ Some clinical articles also suggested that cross-linked ADM is advantageous over non-cross-linked ADM. $^{2,6,8}$ However, vascular ingrowth through collagen degradation and endothelial cell ingrowth into the cross-linked material is typically limited due to its cross-linkage con- struct, especially in the early postoperative period. ${ }^{1,5,24}$ Our findings in this study suggest that seeding ASCs promotes a rapid degree of host cell infiltration and angiogenesis within cross-linked ADM constructs and enhance biocompatibility. This approach may make cross-linked ADM more clinically useful materials and serves as novel method for enhancing incorporation of biomaterials.

Several limitations of our study must be addressed. First, the act of injecting the material with a needle to deliver cells into the interstitium may in fact create structural imperfections within the construct. Further study investigating mechanical performance is needed. Second, although we placed the needle path facing on the top of subcutaneous pocket so as not to make a cell migration pathway through the needle hole from the base, these needle tracks may in fact act as passage ways for cells to infiltrate the material from the top and discount the effect of the ASCs engraftment. This approach may help to improve the biocompatibility of these cross-linked materials by creating a stimuli for deep material ingrowth.

\section{SUMMARY}

ADM can successfully be engrafted with ASCs. Interstitial engraftment of ASCs into ADM via injection enhances regional infiltration of host cells and angiogenesis, whereas 
onlay seeding showed relatively broad and superficial cell infiltration. These findings may be applied to improve the incorporation of avascular engineered constructs. Efforts to deliver ASCs to acellular constructs should focus on directed delivery of the cells to specific regions or zones of the construct. This could be approached by engineering the constructs in a way that is designed to house cells within the interstitium rather than on the surface.

\section{REFERENCES}

1. Jarman-Smith ML, Bodamyali T, Stevens C, Howell JA, Horrocks $\mathrm{M}$, Chaudhuri JB. Porcine collagen crosslinking, degradation and its capability for fibroblast adhesion and proliferation. J Mater Sci: Mater Med 2004;15:925-932.

2. Hsu PW, Salgado CJ, Kent K, Finnegan M, Pello M, Simons R Atabek U, Kann B. Evaluation of porcine dermal collagen (Permacol) used in abdominal wall reconstruction. J Plast Reconstr Surg Nurs 2009:62:1484-1489.

3. Eppley BL. Experimental assessment of the revascularization of acellular human dermis for soft-tissue augmentation. Plast Reconstr Surg 2001;107:757-762.

4. Wong AK, Schonmeyr B, Singh P, Carlson DL, Li S, Mehrara BJ. Histologic analysis of angiogenesis and lymphangiogenesis in acellular human dermis. Plast Reconstr Surg 2008;121:1144-1152.

5. Liang HC, Chang $\mathrm{Y}$, Hsu CK, Lee MH, Sung HW. Effects of crosslinking degree of an acellular biological tissue on its tissue regeneration pattern. Biomaterials 2004;25:3541-3552.

6. Jin J, Rosen MJ, Blatnik J, McGee MF, Williams CP, Marks J, Ponsky J. Use of acellular dermal matrix for complicated ventral hernia repair: Does technique affect outcomes? J Am Coll Surg 2007; 205:654-660.

7. Gaertner WB, Bonsack ME, Delaney JP. Experimental evaluation of four biologic prostheses for ventral hernia repair. J Gastrointest Surg 2007;11:1275-1285.

8. Catena F, Ansaloni L, Gazzotti F, Gagliardi S, Di Saverio S, $D^{\prime}$ Alessandro L, Pinna AD. Use of porcine dermal collagen graft (Permacol) for hernia repair in contaminated fields. Hernia 2007; 11:57-60.

9. Rehman J, Traktuev D, Li J, Merfeld-Clauss S, Temm-Grove CJ, Bovenkerk JE, Pell CL, Johnstone BH, Considine RV, March KL. Secretion of angiogenic and antiapoptotic factors by human adipose stromal cells. Circulation 2004;109:1292-1298.

10. Zuk PA, Zhu M, Mizuno H, Huang J, Futrell JW, Katz AJ, Benhaim $\mathrm{P}$, Lorenz HP, Hedrick MH. Multilineage cells from human adipose tissue: implications for cell-based therapies. Tissue Eng 2001;7: 211-228.
11. Schaffler A, Buchler C. Concise review: adipose tissue-derived stromal cells-basic and clinical implications for novel cell-based therapies. Stem cells 2007;25:818-827.

12. Kim WS, Park BS, Sung JH, Yang JM, Park SB, Kwak SJ, Park JS. Wound healing effect of adipose-derived stem cells: a critical role of secretory factors on human dermal fibroblasts. J Dermatol Sci 2007;48:15-24.

13. Altman AM, Matthias $N$, Yan $Y$, Song $Y H$, Bai $X$, Chiu ES, Slakey DP, Alt EU. Dermal matrix as a carrier for in vivo delivery of human adipose-derived stem cells. Biomaterials 2008;29: 1431-1442.

14. Orbay H, Takami Y, Hyakusoku H, Mizuno H. Acellular dermal matrix seeded with adipose-derived stem cells as a subcutaneous implant. Aesthetic Plast Surg 2011;35:756-763.

15. Prichard HL, Reichert WM, Klitzman B. Adult adipose-derived stem cell attachment to biomaterials. Biomaterials 2007;28: 936-946.

16. O'Brien FJ, Harley BA, Yannas IV, Gibson LJ. The effect of pore size on cell adhesion in collagen-GAG scaffolds. Biomaterials 2005;26:433-441.

17. Thevenot $P$, Nair A, Dey J, Yang J, Tang L. Method to analyze three-dimensional cell distribution and infiltration in degradable scaffolds. Tissue Eng Part C Methods 2008;14:319-331.

18. Schonmeyr B, Clavin N, Avraham T, Longo V, Mehrara BJ. Synthesis of a tissue-engineered periosteum with acellular dermal matrix and cultured mesenchymal stem cells. Tissue Eng Part A 2009;15:1833-1841.

19. Erdag G, Sheridan RL. Fibroblasts improve performance of cultured composite skin substitutes on athymic mice. Burns 2004;30: 322-328.

20. Altman AM, Chiu ES, Bai X, Yan Y, Song YH, Newsome RE, Alt EU. Human adipose-derived stem cells adhere to acellular dermal matrix. Aesthetic Plast Surg 2008;32:698-699.

21. Van Belle E, Witzenbichler B, Chen D, Silver M, Chang L, Schwall R, Isner JM. Potentiated angiogenic effect of scatter factor/hepatocyte growth factor via induction of vascular endothelial growth factor: the case for paracrine amplification of angiogenesis. Circulation 1998;97:381-390.

22. Chavakis $E$, Dimmeler $S$. Regulation of endothelial cell survival and apoptosis during angiogenesis. Arterioscler Thromb Vasc Biol 2002;22:887-893.

23. Fukumura D, Ushiyama A, Duda DG, Xu L, Tam J, Krishna V, Chatterjee K, Garkavtsev I, Jain RK. Paracrine regulation of angiogenesis and adipocyte differentiation during in vivo adipogenesis. Circ Res 2003;93:e88-97.

24. Macleod TM, Williams G, Sanders R, Green CJ. Histological evaluation of Permacol as a subcutaneous implant over a 20-week period in the rat model. Brit J Plast Surg 2005;58:518-532. 